\title{
-Original一
}

\section{Relation of Leukocytosis in Prostatic Fluid and Inflamed Prostatic Tissue}

\author{
Narumi Tsuboi ${ }^{1}$, Taiji Nishimura ${ }^{1}$, Haiwen $\mathrm{Chen}^{2}$, Yoshihiko Norose ${ }^{3}$, \\ Masumi Shimizu ${ }^{3}$, Yukihiro Kondo ${ }^{1}$, Go Kimura ${ }^{1}$ and Yu Fukuda ${ }^{4}$ \\ ${ }^{1}$ Department of Urologic Surgery, Graduate School of Medicine, Nippon Medical School \\ ${ }^{2}$ Department of Urology, Second Hospital, Xi'an Jiaotong University, China \\ ${ }^{3}$ Department of Bioregulatory Science, Graduate School of Medicine, Nippon Medical School \\ ${ }^{4}$ Department of Analytic Human Pathology, Graduate School of Medicine, Nippon Medical School
}

\begin{abstract}
In our continuing investigation of the significance of leukocytosis in prostatic fluid (PF), the relation of leukocytosis in $\mathrm{PF}$ to that in selected sections of prostate with significant inflammation was studies with whole-mount specimens obtained at radical prostatectomy from 12 patients with prostate cancer. Although leukocytosis was observed both in PF and in prostate tissue in all 12 patients, there was no correlation between the leukocyte count in $\mathrm{PF}$ and the intensity of inflammation. However, the ratio of macrophages among leukocytes in PF correlated with the number of ducts filled with macrophages in prostate tissue $(\mathrm{p}=0.0481)$. This finding was consistent with our previous finding that activation of macrophages in PF reflects active inflammation in prostate tissue. Further studies are needed to clarify the roles of macrophages and whole leukocytes in $\mathrm{PF}$ and prostate tissue.
\end{abstract}

(J Nippon Med Sch 2007; 74: 210-216)

Key words: prostatitis, prostatic fluid, macrophages, leukocytes, inflammation

\section{Introduction}

Although leukocytosis in prostatic fluid (PF) has often been described, its significance remains unclear $^{1-8}$. In acute bacterial prostatitis and in nonbacterial prostatitis (NBP), leukocytosis is observed in PF. However, leukocytosis in PF has been observed in most asymptomatic elderly subjects. ${ }^{8}$. Furthermore, benign prostatic hyperplasia is also accompanied by inflammation ${ }^{3}$. In contrast, leukocytosis in $\mathrm{PF}$ is not observed in patients with prostatitis even if the duct from lesion is obstructed.

A study of the relation of leukocytosis in PF to that in selected sections with the most significant inflammation from whole-mount specimens obtained at radical prostatectomy would help clarify leukocytosis in PF. In the present study we examined this relationship in 12 patients with prostatic cancer, with an emphasis on macrophages identified with a monoclonal antibody (mAB).

\section{Patients and Methods}

Twelve consecutive patients with clinically localized prostate cancer who underwent prostatectomy from November 2005 through February 2006 were included in this study. Patient

Correspondence to Taiji Nishimura, Department of Urology, Nippon Medical School, 1-1-5 Sendagi, Bunkyo-ku,

Tokyo 113-8603, Japan

E-mail: ben@nms.ac.jp

Journal Website (http://www.nms.ac.jp/jnms/) 
ages ranged from 60 to 73 years with a median age of 66.4 years. PF was obtained with transrectal digital massage and collected in a tube containing $100 \mu l$ of phosphate-buffered solution (PBS) after induction of general anesthesia just before surgery, and whole-mount radical prostatectomy specimens were obtained from the same patients.

The volume of PF was calculated by subtracting the volume of PBS from the whole volume in the tube after collection of $\mathrm{PF}$, and then the leukocyte count per millimeter was determined with a hemocytometer. The number of leukocytes per milliliter of $\mathrm{PF}$, and the percentages of macrophages among leukocytes after monoclonal staining were determined.

\section{Immunocytology}

Ten thousand leukocytes from PF were made to adhere to glass slides using cytospin and were fixed with an acetone/methanol solution for 10 minutes. The slides were immediately stained in the following order: (1) inactivated for endogenous peroxidase, (2) incubated with $10 \%$ horse serum at room temperature for 1 hour with a Avidin/Biotin Blocking Kit (Vector Laboratories, Burlingame, CA, USA), (3) incubated with a horse anti-human CD68 (Vector Laboratories) at $4^{\circ} \mathrm{C}$ overnight, and (4) incubated with a biotinylated mouse anti-horse IgG for 1 hour at room temperature using a Vecstain ABC Kit (Vector Laboratories).

In macrophages with positive $\mathrm{mAb}$ staining, the cytoplasm appeared brown (Fig. 1). The CD68positive macrophages and other leukocytes counterstained with hemotoxylin and eosin on whole slide were counted under a microscope with a 100square rectangular grid at the center of view. Because the antigens of $\mathrm{T}$ lymphocytes are localized on the cell wall, only the cell wall, and not the cytoplasm, was stained (Fig. 2)

\section{Staining of Whole-mount Specimens}

Whole-mount specimens obtained at radical prostatectomy were sectioned at $4 \mathrm{~mm}$, and routine pathological examination was performed. Additional unstained sections from areas where inflammation was most significant were subjected to immunohistochemical staining with the following method. The section was deparaffinized to water, hydrogen-peroxide-blocked, and stained with an $\mathrm{mAb}$ as indicated in the description of staining of leukocytes adhered to glass slides. The extent of inflammation and inflammatory aggressiveness were scored with the method of Irani et al. ${ }^{9}$ The extent of inflammation was graded with a 4-point scale: 0, no inflammatory cells; 1 , scattered inflammatory cell infiltrate within the stroma without lymphoid nodules; 2, nonconfluent lymphoid nodules; and 3, confluent infiltrate of mononuclear inflammatory cells. Scores for inflammatory aggressiveness were: 0 , no evidence of active prostatitis; 1 , infiltration of polymorphonuclear leukocytes in the epithelium of glands or ducts; 2, infiltration of polymorphonuclear leukocyte microabscess in the lumina of glands or ducts; and 3, granulomatous prostatitis.

Correlations of the number of leukocytes per milliliter of PF and the percentages of macrophages among leukocytes with the scores of the extent of inflammation and inflammatory aggressiveness were investigated. Additionally, the correlation between the percentage of macrophages among leukocytes and the number of ducts filled with macrophages in prostatic tissue was investigated.

\section{Antibodies and Flow Cytometry}

Fluorescein isothiocyanate-conjugated antibodies to CD1a, CD3, and CD19 were purchased from PharMingen (San Diego, CA, USA). Flow cytometric analysis was performed with a FACScan cell sorter (Becton Dickinson, Mountain View, CA, USA). Fluorescein isothiocyanate-labeled mAbs were added to the pellet and incubated for 30 minutes at $4{ }^{\circ} \mathrm{C}$. Cells were washed twice and resuspended with 0.4 $\mathrm{m} l$ of PBS containing $0.01 \%$ sodium azide and $0.15 \%$ bovine serum albumin for acqusition. Data were analyzed with CellQuest software (Becton Dickinson) as previously described ${ }^{10}$.

Statistical analysis was performed with Student's $t$-test. In all cases the level of significance was accepted as $\mathrm{p}<0.05$.

Informed consent for obtaining $\mathrm{PF}$ and for use of $\mathrm{PF}$ and resected whole-mount prostatectomy specimens for possible investigation was obtained 
Table 1 Background characteristics of all 12 patients and findings

\begin{tabular}{cccc}
\hline Case No. & $\begin{array}{c}\text { Leukocytes } \\
\text { number/ml in PF }\end{array}$ & $\begin{array}{c}\text { Ratio of macrophages } \\
\text { among leukocytes in PF }\end{array}$ & $\begin{array}{c}\text { Number of ducts } \\
\text { filled with macrophages }\end{array}$ \\
\hline 1 & $2.37 \times 10^{7}$ & $16.2 \%$ & 3 \\
2 & $3.92 \times 10^{6}$ & $3.6 \%$ & 3 \\
3 & $3.58 \times 10^{6}$ & $44.5 \%$ & 19 \\
4 & $1.52 \times 10^{7}$ & $27.2 \%$ & 3 \\
5 & $3.05 \times 10^{5}$ & $9.3 \%$ & 8 \\
6 & $1.94 \times 10^{6}$ & $57.5 \%$ & 11 \\
7 & $9.20 \times 10^{6}$ & $3.7 \%$ & 3 \\
8 & $3.92 \times 10^{6}$ & $9.4 \%$ & 4 \\
9 & $3.12 \times 10^{6}$ & $5.2 \%$ & 4 \\
10 & $2.24 \times 10^{6}$ & $6.7 \%$ & 0 \\
11 & $4.16 \times 10^{6}$ & $7.6 \%$ & 4 \\
12 & $3.44 \times 10^{6}$ & $20.1 \%$ & 28 \\
\hline
\end{tabular}

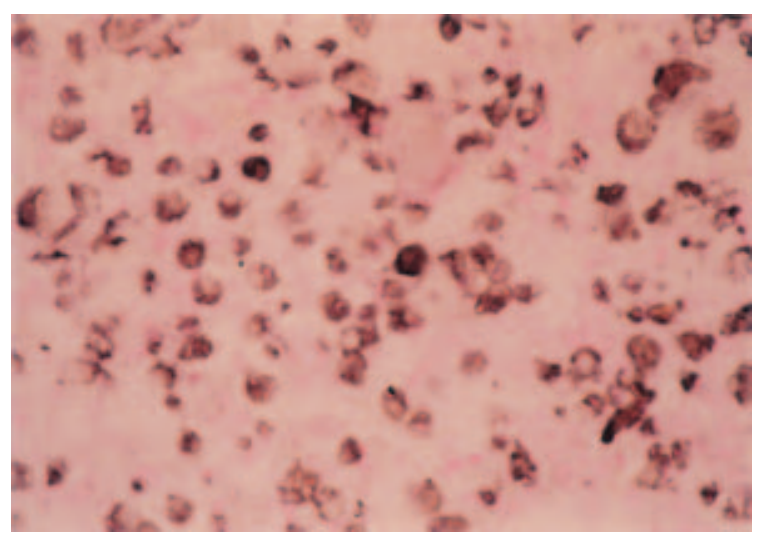

Fig. 1 mAb staining of macrophages in PF (brown cytoplasma). Reduced from $\times 400$.

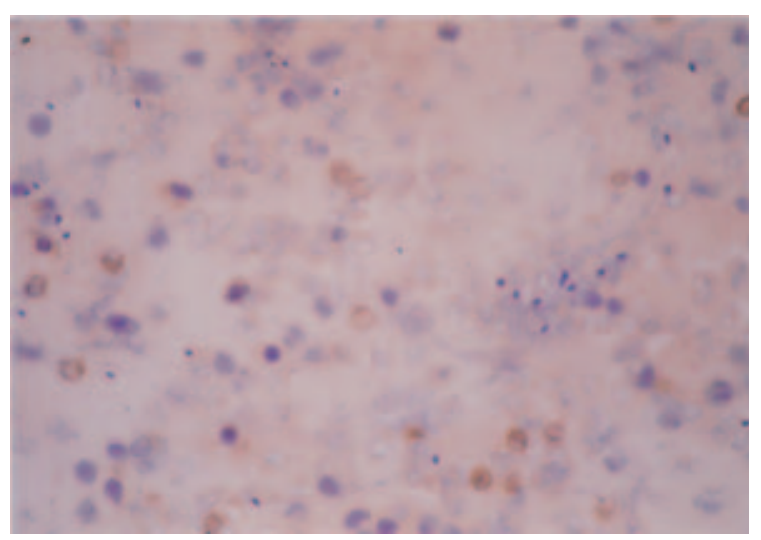

Fig. $2 \mathrm{mAb}$ staining of $\mathrm{T}$ lymphocytes in $\mathrm{PF}$ (browncell wall). Reduced from $\times 400$.

from each patient before prostatectomy.

\section{Results}

Background characteristics of all patients and the

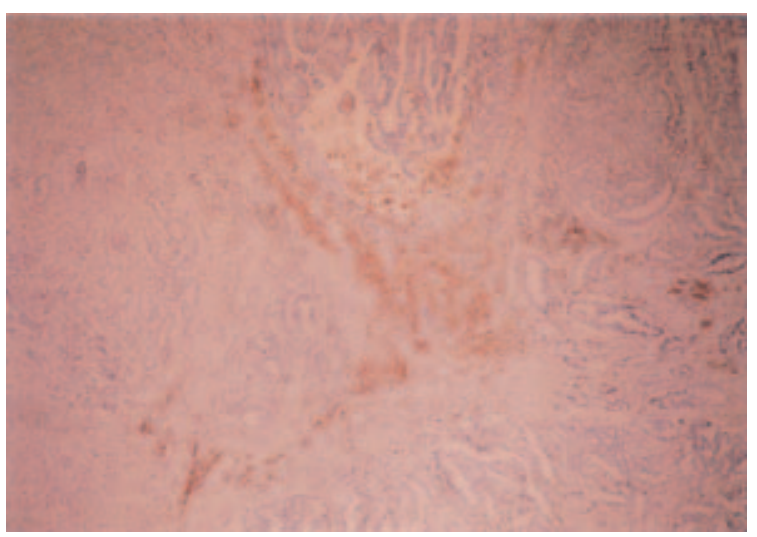

Fig. 3 Macrophages appeared in the interstitial tissue of the prostate in sections of wholemount prostate specimens. Reduced from $\times 40$.

results are shown in the Table 1 . The mean $( \pm \mathrm{SD})$ interval between prostate biopsy and prostatectomy was $86.25 \pm 36.45$ days. The mean $( \pm \mathrm{SD})$ number of leukocytes per milliliter of PF was $6.22 \pm 6.76 \times 10^{6}$. All patients demonstrated prostatic inflammatory infiltrate, and the extent of inflammation was grade 1 in 1 patient (8.3\%) and grade 2 in 11 patients (91.7\%). When the scoring method proposed by Irani et al. for the extent of inflammation ${ }^{9}$ was applied to macrophages (Fig. 3), the findings were entirely consistent with those described above.

Inflammatory aggressiveness was grade 1 in 2 patients (16.7\%) and grade 2 in 10 patients (83.3\%). There was no correlation between the leukocyte count in $\mathrm{PF}$ and the extent of inflammation or the inflammation aggressiveness $(\mathrm{p}=0.4311$ and $\mathrm{p}=0.1845$, respectively). 


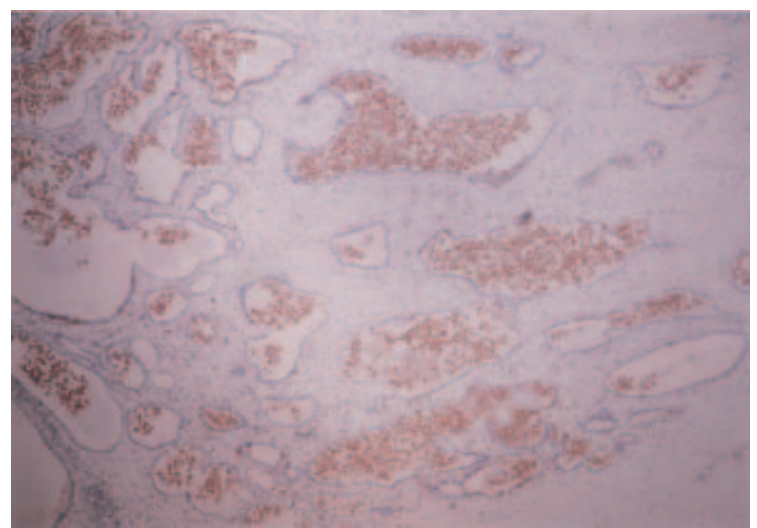

Fig. 4 Many prostate ducts were filled with macrophages in sections of whole-mount prostatectomy specimens with the most significant inflammation . Reduced from $\times 40$.

The average percentage of macrophages among leukocytes was $12.2 \pm 12.56 \%$. Cases with as many as 19 or 28 ducts filled with macrophages (Fig. 4) tended to show higher percentages of macrophages, and these two findings were correlated $(p=0.0410)$.

\section{Discussion}

Many researchers have studied the significance of leukocytes in $\mathrm{PF}$ in relation to inflammation in the prostate $^{1-8}$. Leukocytosis in PF has been observed in most asymptomatic elderly subjects. Furthermore, benign prostatic hyperplasia is also accompanied by inflammation ${ }^{3}$. Our recent study confirmed leukocytosis in PF with an average leukocyte count of $4.86 \pm 6.07 \times 10^{6} / \mathrm{ml}$ in elderly patients without symptoms of urinary tract infection who underwent prostate biopsy because of an elevated serum level of prostate-specific antigen ${ }^{8}$. In contrast, leukocytosis in $\mathrm{PF}$ is not observed in patients with prostatitis even if the duct from the lesion is obstructed ${ }^{4}$. Obstruction of ducts with corpora amylacea and macrophages was observed in the present study.

In the present study, we studied the significance of leukocytosis in PF and examined leukocytosis both in PF and in whole-mount prostate specimens from the same patients. To our knowledge, no previous papers have described the relation of leukocytosis in PF and in areas of inflamed prostatic tissue from the same patient, especially not with

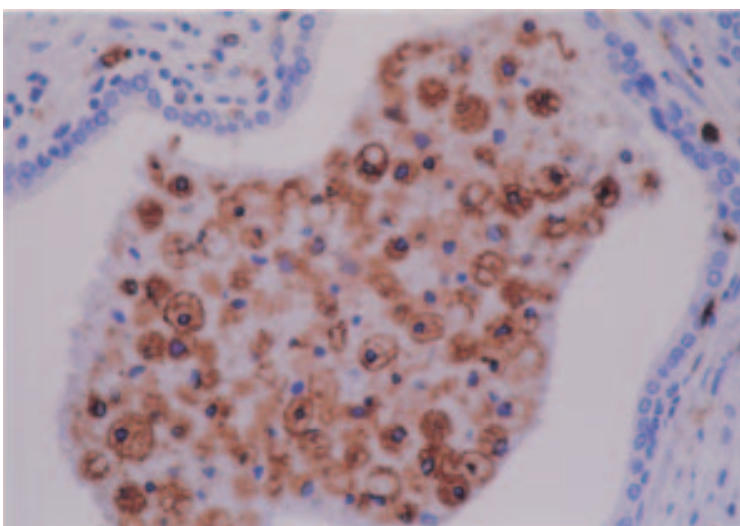

Fig. 5 Macrophages and polymorphonuclear leukocytes in prostate ducts surrounded by amorphous materials in sections of wholemount prostate specimens. Reduced from $\times 400$.

$\mathrm{mAb}$ staining. Initially, we attempted to examine three leukocyte subpopulations: macrophages, $\mathrm{T}$ lymphocytes, and B lymphocytes. Ludwig et $\mathrm{al}^{5}$ has successfully performed immunocytological staining with mAbs of these leukocyte subpopulations in urine after prostatic massage in patients with chronic prostatitis. However, we found that immunocytological staining of $\mathrm{T}$ and $\mathrm{B}$ lymphocytes in PF was quite difficult, although staining was successful in a few cases in a preliminary study (Fig. 2). The reason findings differed between the series of Ludwig et $\mathrm{al}^{5}$ and our series may have been differences in materials and patients. That is, we used PF from patients who had cancer without clinical symptoms of prostatitis, whereas Ludwig et $\mathrm{al}^{5}$ used urine after prostatic massage instead of PF from patients with chronic prostatitis. Leukocytes in PF are often surrounded by amorphous materials that may prevent staining with mAbs (Fig. 5). Urokinase in urine may help dissolve amorphous materials surrounding leukocytes and consequently facilitate staining with mAbs. Neither the addition of heparin to PBS in which PF was collected to prevent leukocyte aggregation nor the washing of PF with PBS improved the quality of immunocytological staining. Therefore, flow cytometry was performed after immunocytological staining of $\mathrm{T}$ and $\mathrm{B}$ lymphocytes in 2 patients. This procedure was performed by a researcher with significant experience in this area (M.S.) ${ }^{10}$ We found 

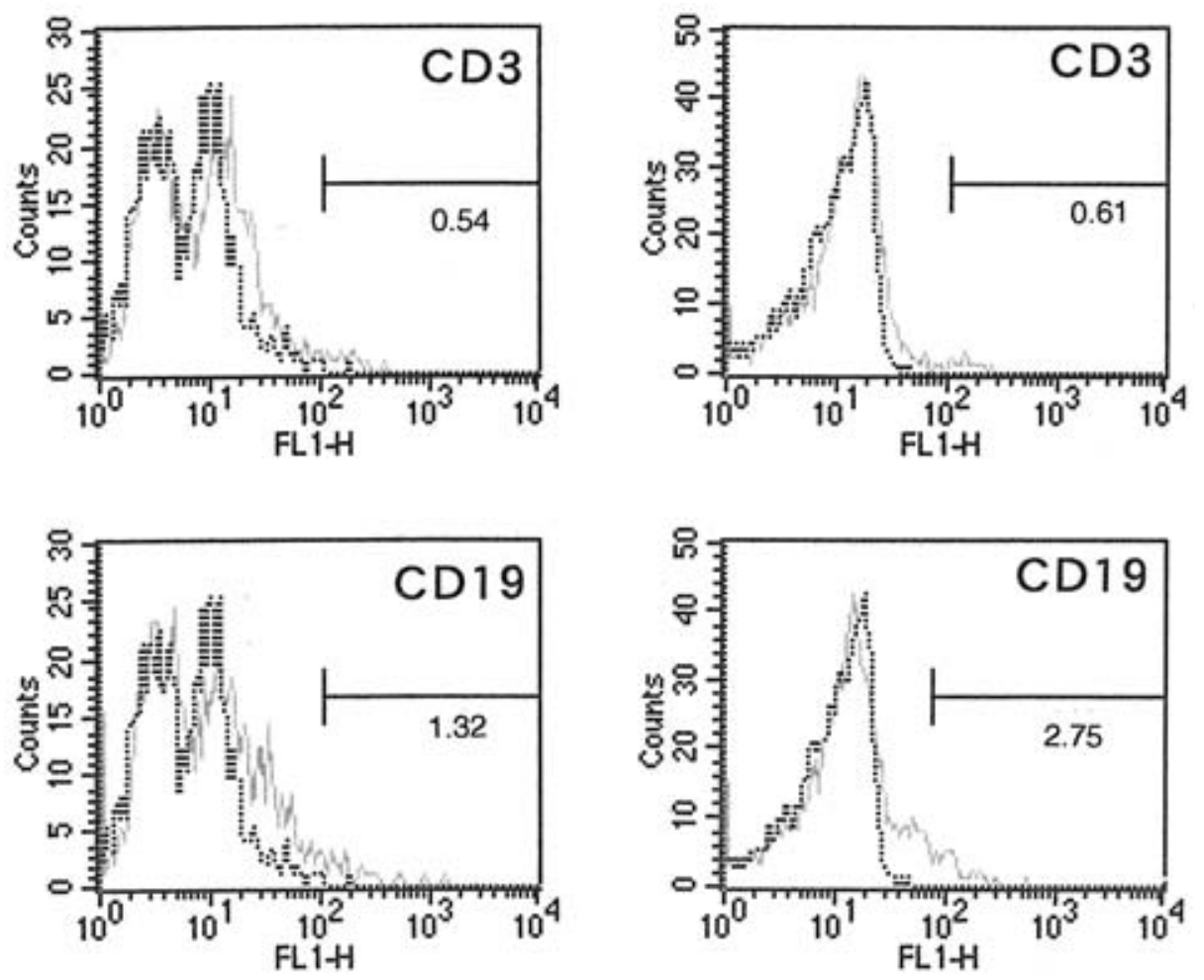

Fig. 6 Phenotype of leukocytes in PF. CD3 (upper panel) and CD19 (lower panel) in two patients who underwent prostate biopsy with serum levels of prostate-specific antigen of 10.0 and $36.0 \mathrm{ng} / \mathrm{ml}$, respectively, in a preliminary experiment.

that both lymphocyte populations comprised quite low percentages: $0.54 \%$ and $0.61 \%$ for $\mathrm{T}$ lymphocytes, and $1.32 \%$ and $2.75 \%$ for $\mathrm{B}$ lymphocytes (Fig. 6). Muller et $\mathrm{al}^{6}$ described $\mathrm{mAb}$ staining of expressed prostatic secretions (EPSs) as unpublished data. That Muller et al have not published newer papers concerning $\mathrm{mAb}$ staining of EPSs may suggest difficulty with such staining.

Finally we decided to observe only CD68-positive macrophages as a leukocyte subpopulation in the present study because we thought we could study the relation between leukocytosis in PF and that in prostatic tissue in the same patients by observing only macrophages. However, we found no quantitative correlation between the leukocyte count and the extent of inflammation or inflammation aggressiveness in prostate tissue. However, leukocytosis was observed in both $\mathrm{PF}$ and prostate tissue in all 12 cases. The number of leukocytes in $\mathrm{PF}$ in the present study $\left(6.22 \times 10^{6} / \mathrm{ml}\right)$ was 1.28 times that in our previous study $\left(4.86 \times 10^{6} / \mathrm{ml}\right)$ in patients who underwent prostate biopsy. This difference might be attributed to the effect of biopsy in our previous study.

We found a correlation between the percentage of macrophages among leukocytes in $\mathrm{PF}$ and the number of ducts filled with macrophages (Fig. 4) in areas of prostate tissue with the most significant inflammation $(\mathrm{p}=0.0410)$. For example, in two cases showing as many as 19 or 28 ducts filled with macrophages, the percentage of macrophages to all leukocytes in $\mathrm{PF}$ was as high as $44.5 \%$ and $20.1 \%$, respectively. The average number of ducts filled with macrophages was 7.5 per section with the most significant inflammation in each of the 12 patients. We have previously reported the relation between active NBP and the participation of activated macrophages in PF, expressed by a high percentage of macrophages to leukocytes and a high percentage of adherent and spreading macrophages on glass when these cells were cultured ${ }^{11}$. In 2 patients with a high percentage of macrophages, many macrophages adhered and spread in short-term culture. 

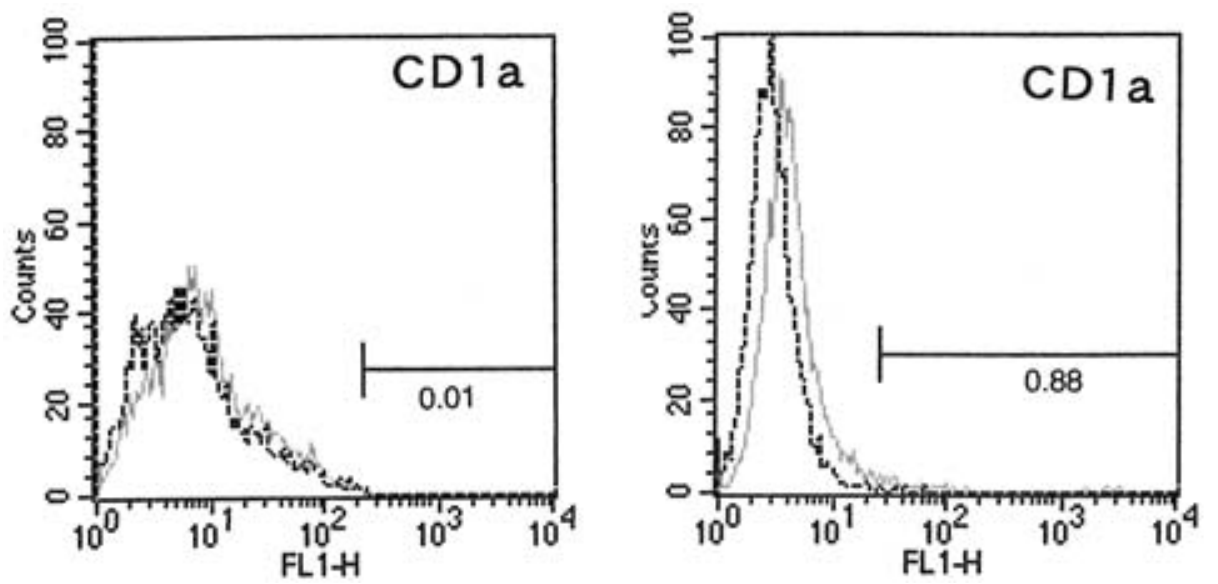

Fig. 7 Phenotype of leukocytes in PF. CDla in two patients with acute bacterial prostatitis in a preliminary experiment.

Ludwig et $\mathrm{al}^{5}$ concluded with quite rough consideration that the increases in macrophages, $\mathrm{T}$ lymphocytes, and $\mathrm{B}$ lymphocytes in prostatic secretions indicate the participation of both the cellular and humoral immune systems in the inflammatory process. Recently dendritic cells (DCs) instead of macrophages have been considered antigen-presenting cells, and Steinman et $\mathrm{al}^{12}$ have indicated that macrophages are located far from the site of antigens and that macrophages play a role in lower tissue reaction through cytokine production rather than a role in antigen presentation. We are interested in the role of DCs in prostatitis and have attempted to study, by means of fluorescenceactivated cell sorting, the percentage of DCs in PF from patients with prostatitis; however, in a preliminary study percentages of DCs detected with CDla were low at $0.47 \%$ and $0.07 \%$ in 2 cases (Fig. 7). Therefore, we discontinued the study of DCs in PF because only a small number of DCs could be obtained and because at least $1 \times 10^{6} \mathrm{DCs}$ in each case are necessary for immunocytological examination. Concerning the role of macrophages, we have been studying macrophages in PF for 30 years; however, we have obtained only a few findings: 1) the dominant participation of macrophages in the early stage of prostatitis compared with the chronic stage $\left.\mathrm{e}^{13} ; 2\right)$ this dominant participation of macrophages lasts a few months in $30 \%$ of cases of $\mathrm{NBP}^{14}$; 3) macrophages in the early stage of NBP are more activated than are macrophages in the chronic stage ${ }^{11}$; 4 ) the longevity of macrophages in PF is increased with the addition of phosphatidylserine to culture medium $^{15}$; 5) the longevity of perpheral blood monocytes is increased with addition of autogenic or allogenic activated macrophages in $\mathrm{PF}$ from patients with $\mathrm{NBP}^{16}$; 6) the low level of interleukin-1ra in relation to that of interleukin-1 secreted in PF is unlikely to cause prolongation of $\mathrm{NBP}^{17}$; and 7) the expression of antiapoptotic Bcl-2 on leukocytes in PF is not a cause of leukocyte longevity in long-term prostatitis? We believe that many more studies will be needed to clarify the role of macrophages in prostate tissue and PF.

\section{Conclusion}

Although patients included in the present study did not demonstrate symptoms of prostatitis, this is the first study to compare leukocytosis in $\mathrm{PF}$ with that in areas of inflamed prostatic tissue in the same patient, with a special emphasis on macrophages identified with an mAb. Leukocytosis was observed both in $\mathrm{PF}$ and in prostate tissue in all 12 cases; however, there was no correlation between the leukocyte count in PF and the intensity of inflammation in prostate tissue.

We found a correlation between activation of macrophages in $\mathrm{PF}$, expressed as a high percentage of macrophages among leukocytes, and the number of ducts filled with macrophages in prostate tissue 
where the inflammation was most significant. This finding was compatible with the results of our previous study, which found that activation of macrophages in $\mathrm{PF}$ reflects active inflammation in prostate tissue.

\section{References}

1. Ghormley KO, Cook ED, Needham GM: Bacterial flora in chronic prostatitis. Amer J Clin Path 1954; 24: 186-193.

2. O'Shaugnessy EJ, Parrino PS, White JD: Chronic prostatitis-fact or fiction. JAMA 1956; 160: 540-542.

3. Perez-Blas M, Martinez-Martin B, Carballido J, et al: Defective functional response to membrane stimuli in lymphocytes from patients with benign prostatic hyperplasis. Clin Exp Immunolo 1995; 101: 521-526.

4. Blacklock NJ: Anatomical factors in prostatitis. Br J Urol 1974; 46: 47-54.

5. Ludwig M, Steltz C, Huwe P, Schfter R, Altmannsberger $\mathrm{M}$, Weidner $\mathrm{N}$ : Immunocytological analysis of leukocyte subpopulations in urine specimens before and after prostatic massage. Eur Urol 2001; 39: 277-282.

6. Muller $\mathrm{CH}$, Berger $\mathrm{RE}, \mathrm{Mohr}$ LE, Krieger JN: Comparison of microscopic methods for detecting inflammation in expressed prostatic secretions. J Urol 2001; 39: 2518-2524.

7. Abe H, Nishimura T, Asoh S, Ohta S: Expression of Bcl-2 on leukocytes in prostatic fluid from patients with acute and chronic prostatitis. J Infect Chemother 2003; 9: 156-159.

8. Chen H, Tsuboi N, Nishimura T, et al.: Study of prostatic fluid from patients with elevated levels of prostate-specific antigen. J Nippon Med Sch 2006; 73: $24-28$.

9. Irani J, Levellain $\mathrm{P}$, Goujon JM, Bon D, Dore B, Aubert J: Inflammation in benign prostatic hyperplasia: correlation with prostatic specific antigen value. J Urol 1997; 157: 1301-1303.

10. Ishii R, Shimizu M, Nakagawa Y, Shimizu K, Tanaka $\mathrm{S}$, Takahashi $\mathrm{H}$ : In vivopriming of natural killer $\mathrm{T}$ cells by dendritic cells pulsed with hepatoma-derived acid-eluted substances. Cancer Immunol Immunother 2004; 53: 383-390.

11. Nishimura $T$, Terashima $Y$, Hattori $T$, Satoh $M$, Yoshida K, Akimoto M: Study of macrohpages in prostatic fluid from nonbacterial prostatitis patients. V. Relation between activation of macrophages and stage of prostatitis. Uro Int 1991; 46: 15-17.

12. Steinman RM, Hawiger D, Liu K, et al: Dendritic cell function in vivo during the steady state: a role in peripheral tolerance. Ann NY Acad Sci 2003; 987: $15-25$.

13. Nishimura T, Kanamori S, Akimoto M, Kawai H: Macrophages in prostatic fluid. Br J Urol 1980; 52: 381-385.

14. Nishimura T, Kanamori S, Yoshida K, Akimoto M, Kawai H: Longitudinal study of macrophages in prostatic fluid from nonbacterial prostatitis patients. Urology 1981; 18: 255-257.

15. Nishimura T, Terashima $Y$, Hattori $T$, et al:: The survival rate of macrophages in prostatic fluid from nonbacterial prostatitis patients. J Nippon Med Sch 1992; 59: 274-275.

16. Nishimura T, Satoh M, Terashima Y, et al: The role of macrophages in prostatic fluid in prolongation of nonbacterial prostatitis. J Nippon Med Sch 1994; 61: 59-61.

17. Nishimura $T$, Abe $H$, Ito $H$, Ikeda $K$, Oka $F$, Yamamoto M: IL-1ra versus IL-1 levels in prostatic fluid from prostatitis patients. Uro Int 1998; 60: 9296.

(Received, January 5, 2007)

(Accepted, February 13, 2007) 